\title{
Obésité, un Journal pour demain
}

\author{
S. Czernichow $\cdot$ J. Dargent \\ C) Springer-Verlag France 2010
}

Pourquoi se battre pour défendre un journal francophone dédié à l'obésité ? Pourquoi ne pas renoncer et abandonner son contenu à des revues anglophones prestigieuses ou bien à des revues reconnues qui prennent en compte les maladies métaboliques, la nutrition ou le diabète? En d'autres termes, existe-t-il encore une place pour une revue sur ce créneau que d'aucuns jugent étroit?

Nous répondons oui sans hésitation:

- parce que chirurgiens et médecins sont désormais partenaires et articulent leurs positions. Si l'on ne devait retenir qu'un exemple, c'est celui de la compréhension progressive des mécanismes de la satiété qui interagissent avec certaines interventions comme le by-pass gastrojéjunale : la mise en évidence de réponses entérohormonales spécifiques est riche de promesses thérapeutiques, comme le prouvent les essais actuels sur le PYY et le GLP-1 ;

- parce qu'en matière d'obésité, des solutions techniques sont sur la table et ne cessent d'évoluer;

- parce que de nouvelles perspectives émergent dans la recherche médicamenteuse, génétique, épidémiologique et dans les sciences sociales ;

- parce que la spécialité médicale « obésité » francophone a besoin d'un espace propre, qui ne soit pas seulement l'annexe des autres volumineux sujets que sont la nutrition au sens plus général, les dyslipidémies ou le diabète, tant l'ampleur du phénomène épidémiologique est importante ;

- parce que cet espace est davantage partagé que par le passé. Après bien des questionnements, les chirurgiens digestifs ont trouvé le leur, mais l'avenir des techniques bariatriques ne leur appartient pas exclusivement. Nous avons l'habitude de vivre autour d'une distinction chirur-

gie restrictive-malabsorptive depuis plus de 30 ans, opposant schématiquement des techniques qui ne peuvent « durer » sans encadrement hygiénodiététique et d'autres qui, au moins pour partie, jouent sur la diminution de l'absorption des aliments. Des évolutions techniques rapides brouillent cette distinction. La plus importante est probablement le fait que, l'objectif mini-invasif étant désormais acquis, l'horizon à court terme est celui des « techniques bariatriques non invasives » qui seront partagées avec la gastroentérologie. Notre ambition sera alors double :

- améliorer l'intensité et surtout la longévité de la perte de poids et l'efficacité de résolution des comorbidités ;

- affiner le compromis sous-jacent à tout traitement de l'obésité, le ratio bénéfices/risques, et permettre de relever nos présents défis, c'est-à-dire de traiter la frange de population des patients obèses sévères hors indication chirurgicale actuelle ; traiter son cœur de cible ( $90 \%$ des patients avec IMC $>40 \mathrm{~kg} / \mathrm{m}^{2}$ lui échappent finalement).

Même si les groupes les plus favorisés de la population française sont moins touchés que les plus précaires, même si la prévalence n'a pas augmenté en dix ans dans les catégories sociales les plus élevées, l'obésité continue de concerner l'ensemble de la population. En dehors de l'approche médicochirurgicale, les mesures de santé publique doivent se développer et s'optimiser pour enrayer l'épidémie qui touche près de 6,5 millions de personnes en France.

Enfin, pour conclure, nous tenons à remercier chaleureusement le précédent rédacteur en chef, le Pr Olivier Ziegler, pour le travail remarquable qu'il a accompli en animant et en développant la revue depuis son début.

\footnotetext{
S. Czernichow $(\bowtie)$

Faculté SMBH, 74, rue Marcel-Cachin,

F-93017 Bobigny, France

e-mail : czernichow@uren.smbh.univ-paris13.fr

J. Dargent $(\bowtie)$

Polyclinique Lyon-Nord de Rillieux,

941, rue du Capitaine Julien,

F-69140 Rillieux, France

e-mail : jerome.dargent@polyclinique-rillieux.fr
} 